\title{
The Role of Three-dimensional Ultrasound in the Assessment of Congenital Uterine Anomalies
}

\author{
Sarah Tabi, Sanja Kupesic Plavsic
}

\begin{abstract}
Three-dimensional (3D), two-dimensional (2D) ultrasound and saline infusion sonography (SIS) are beneficial tools in diagnosing congenital uterine anomalies. This article illustrates six various case scenarios based on 3D ultrasound images in order to review the concepts of ultrasound diagnosis of congenital uterine anomalies and apply it to the presented case studies. These cases scenarios educate the reader on arcuate uterus, septate uterus, bicornuate uterus, didelphic uterus, uterus duplex and unicornuate uterus. This article also presents the sequence of embryologic events leading to the congenital uterine anomalies. The role of 3D ultrasound in diagnosis and treatment of congenital uterine anomalies is presented, along with its advantages over 2D ultrasound, SIS, X-ray hysterosalpingography, magnetic resonance imaging, hysteroscopy and laparoscopy. The effects of hysteroscopic metroplasty on fertility are also illustrated and discussed. After this case-based discussion is completed, the readers will be able to make a differential diagnosis of the different types of congenital uterine anomalies.
\end{abstract}

Keywords: Three-dimensional ultrasound, Congenital uterine anomalies, Hysteroscopic metroplasty, Fertility, Coronal plane, Surface rendering.

How to cite this article: Tabi S, Kupesic Plavsic S. The Role of Three-dimensional Ultrasound in the Assessment of Congenital Uterine Anomalies. Donald School J Ultrasound Obstet G ynecol 2012;6(4):415-423.

\section{Source of support $\mathrm{Nil}$}

Conflict of interest: None declared

\section{INTRODUCTION}

Unfortunately, the exact epidemiology of congenital uterine anomalies is unclear because it does not often get diagnosed; this is especially the case when the patient is asymptomatic. Reproductive implications and adverse pregnancy outcomes (recurrent pregnancy loss, infertility, spontaneous abortions, abnormal fetal position and preterm labor) vary and depend on a type of the congenital uterine defect. Congenital uterine anomalies are a result of abnormalities that occur along different stages of embryological development. ${ }^{1-3} A$ genesis of müllerian ducts, precursors to the cervix, the uterus, upper two-thirds of the vagina and the fallopian tubes, in the initial stages of development lead to unicornuate uterus. Failure or improper lateral fusion of the müllerian ducts can lead to formation of bicornuate or didelphic uteri. Persistence of the midline septum after lateral fusion of the müllerian ducts causes septate uterus or arcuate uterus. A rcuate uterus is generally asymptomatic and surgery is not indicated for its repair because it usually does not affect pregnancy progression and outcome. The septate uterus is commonly associated with pregnancy complications and hysteroscopic metroplasty is indicated for septal removal.

The reported prevalence in the general population of reproductive age women is 3 to $4 \% .{ }^{1} \mathrm{~A}$ recent study that used 3D ultrasound for diagnosis, reported that the rate of congenital uterine anomalies is $13.3 \%$ in the subfertile female population. ${ }^{2}$ One of the few indications that this abnormality is present in a patient is that the miscarriage rate is higher in women with congenital uterine anomalies compared with healthy women. This condition is uncommonly diagnosed because of the different types of imaging used, which has been traditionally quite limited. This is why the emergence of 3D ultrasound is essential to diagnosis of congenital uterine anomalies, because of its capabilities of imaging the internal and external surfaces of the uterus. Data from recent study indicate that congenital uterine anomalies are highly prevalent in women seeking assisted reproductive treatment and apparently the most common type of anomaly was arcuate uterus and septate uterus. $^{2-4}$

3D ultrasound is highly accurate due to its ability to visualize the uterine cavity and external contours of the uterine myometrium and fundus, which establishes its relevance as a gold imaging method to detect congenital uterine anomalies (Fig. 1). B ecause of multiplanar imaging and surface rendering, the 3D ultrasound can detect uterine anomalies that are more inconspicuous, such as arcuate uterus, which can be overlooked by invasive procedures, like hysteroscopy. A nother advantage is that the 3D ultrasound can group the congenital uterine anomalies into subclassifications. In a study which was performed to investigate the diagnostic accuracy and precision of this novel imaging technique, a $93 \%$ positive and $100 \%$ negative predictive values were confirmed. ${ }^{4}$

Despite the numerous benefits of 3D ultrasound, there are a few limitations as well. A pparently, the 3D ultrasound is not helpful for patients with an endometrium less than $5 \mathrm{~mm}$ thick. The 3D ultrasound shares the same foundation as $2 \mathrm{D}$ ultrasound. Therefore, it shares some of the same shortcomings too. For example, the contrast source used in $2 \mathrm{D}$ ultrasound is the same in $3 \mathrm{D}$ ultrasound, thereby having 


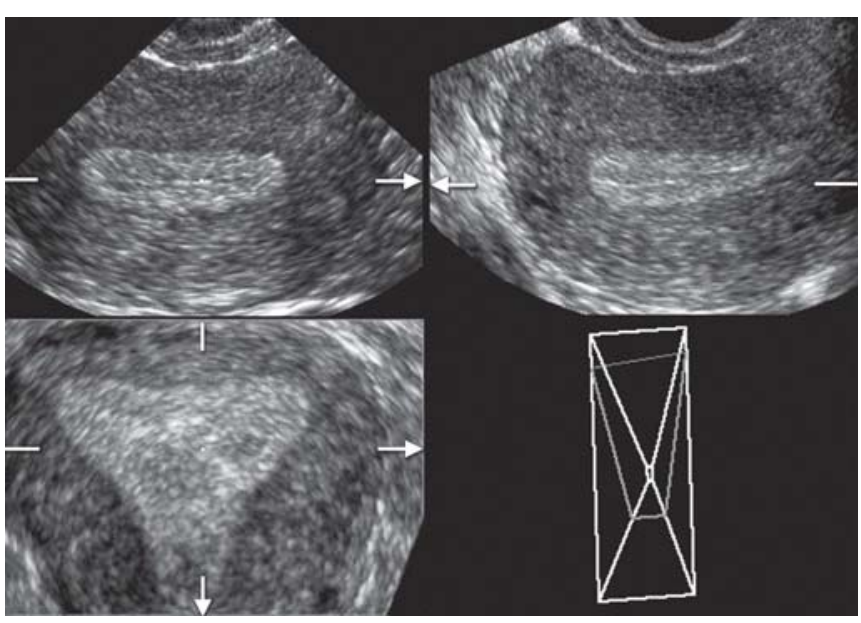

Fig. 1: Three-dimensional ultrasound of the normal uterine cavity. Note: The secretory appearance of the endometrium and triangular shape of the uterine cavity in frontal reformatted section (low left image). Uterine fundus is convex

similar issues of less clarity in the visual image. It is reported that the orientation of the image is not easily determined due to the fact that there is no available 'standardized display convention' because user interfaces are so intricate. ${ }^{4,5}$

\section{CASE REPORTS}

\section{Case 1}

A 22-year-old female (GOPO) comes to your office for her first well woman exam. She has a 5-week history of a constant dull ache of her lower abdomen in her left side that is progressively worsening. Her last menstrual period was 2 weeks ago and it was normal. The patient has regular menstrual cycles, they occur every 28 days with the duration

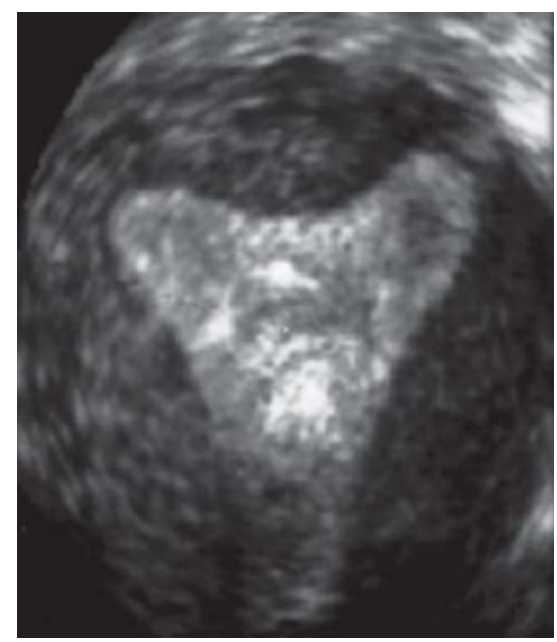

Fig. 2: Three-dimensional (3D) ultrasound and frontal reformatted section of an arcuate uterus (incidental finding). Note: The convex external uterine contour and a slight indentation of the uterine cavity. Arcuate uterus develops due to the failure of resorption of the midline uterine septum. Compare the image with $3 \mathrm{D}$ of the normal uterine cavity (Fig. 1) of 3 to 4 days. Onset of her menarche was at 12 years, age of first intercourse was at 18 years, with no history of sexually transmitted diseases. Her past medical history is unremarkable. She is currently not taking any medications. Her vital signs are normal. Her pregnancy test is negative. Her physical examination was significant for left lower quadrant tenderness and a left adnexal mass and tenderness. She is diagnosed with a functional ovarian cyst of the left ovary and she was scanned to confirm it. Figure 2 illustrates the patient's ultrasound findings. The outer contours of the uterus were normal but there was evidence of a slight indentation of the uterine cavity. This is an incidental finding indicative of an arcuate uterus.

\section{Case 2}

A 33-year-old female (G2P0020) presents to your office with history of recurrent pregnancy loss at 15 and 20 weeks' gestation. She has had two miscarriages within the past 3 years. She is currently in a monogamous relationship with her husband and she wants to have a child. Her menstrual cycles are regular (28-30 days). Her history is significant for dysmenorrhea and premenstrual syndrome. Onset of menarche was at 14 years, age of first intercourse was 20 years, with no history of sexually transmitted diseases. She is currently not on any medications. Her vital signs are blood pressure $120 / 75$, pulse 80 beats per minute, respiratory rate 20 breaths per minute and temperature is $37^{\circ} \mathrm{C}\left(98.6^{\circ} \mathrm{F}\right)$. Her physical examination was normal. Figure 3 illustrates her ultrasound findings.
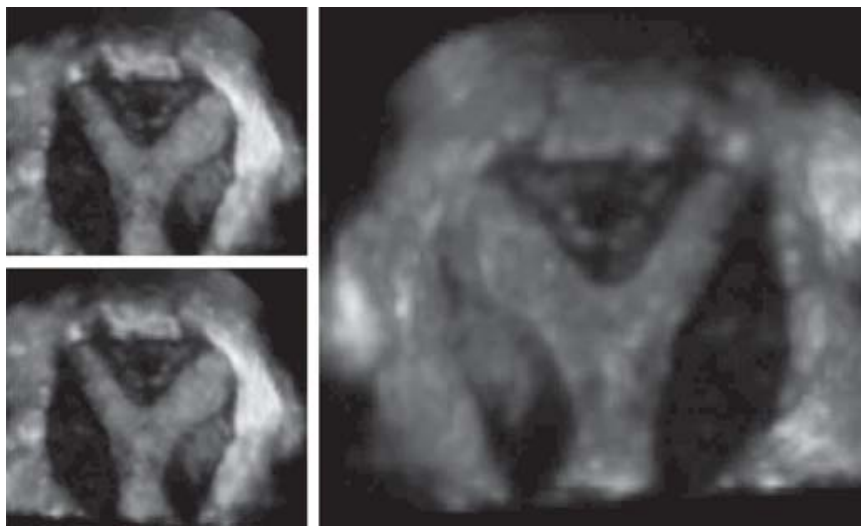

Fig. 3: Three-dimensional ultrasound (frontal reformatted section) of a septate uterus. Note: The clear division of the uterine cavity in the upper half of the uterine cavity. Septate uterus is the most common müllerian duct anomaly and composes approximately $55 \%$ of the uterine anomalies. ${ }^{4,5} \mathrm{Partial}$ septate uterus results from partial failure of resorption of the uterovaginal septum after fusion of the paramesonephric ducts 


\section{Case 3}

A 28-year-old female (G3P0120) presents to your office with recurrent pregnancy loss at 8 and 9 weeks' gestation. Her last pregnancy was a preterm delivery at 25 weeks gestation. Patient is currently sexually active and is in a monogamous relationship with her boyfriend. Onset of menarche was at 11 years, age of first intercourse was 17, with no history of sexually transmitted diseases. Her menstrual cycles occur regularly every 26 to 30 days. She is not taking any medications, only pain medication for relief of her menstrual pain. The patient's history is significant for dysmenorrhea, dyspareunia and chronic pelvic pain. Her vital signs and physical examination are normal. Hysteroscopy and laparoscopy confirmed septate uterus. At the time of combined endoscopic assessment, it was discovered that the patient has endometriosis. Figure 4 reveals her ultrasound findings.
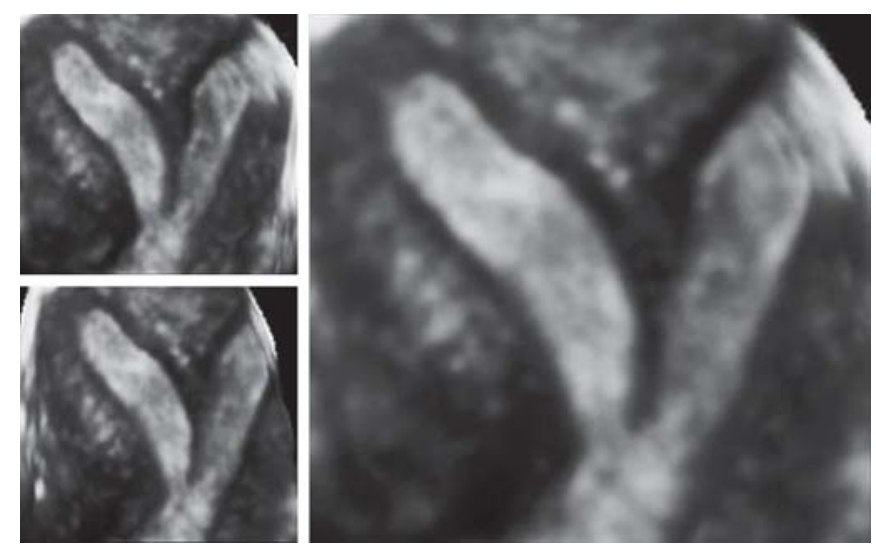

Fig. 4: Three-dimensional ultrasound of the septate uterus. Note: The complete division of the uterine cavity and planar shape of the uterine fundus. Complete septate uterus results from failure of resorption of the uterovaginal septum after fusion of the paramesonephric ducts

\section{Case 4}

A 32-year-old female (G3P0030) presents to your office with history of recurrent pregnancy loss at 7, 8 and 10 weeks gestation. She is currently sexually active and in a monogamous relationship with her husband. Onset of menarche was at 13 years, age of first intercourse was 18, with no history of sexually transmitted diseases. Her menstrual cycles are regular (28-day cycle) with evidence of dysmenorrhea. The patient has no significant medical history. She is not taking any medications. Her vital signs are blood pressure 115/70, pulse 80 beats per minute, respiratory rate 20 breaths per minute and temperature is $37^{\circ} \mathrm{C}\left(98.6^{\circ} \mathrm{F}\right)$. Her physical examination was normal. Figure 5 illustrates her ultrasound findings.

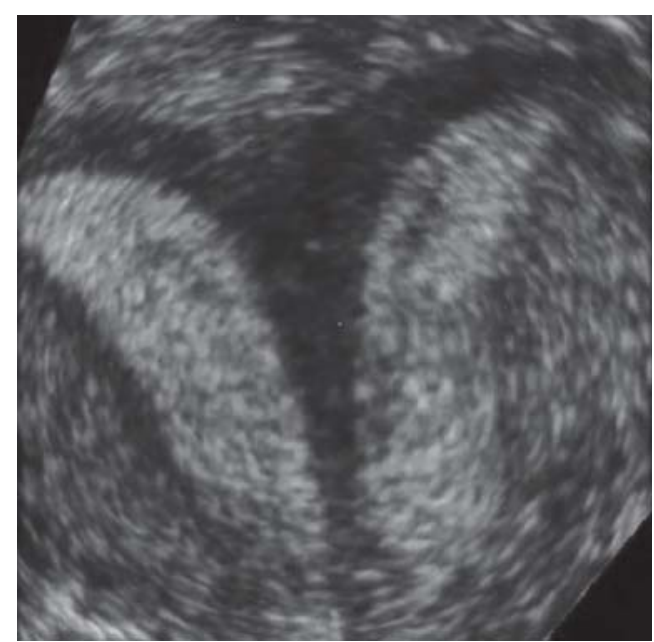

Fig. 5: Three-dimensional ultrasound (frontal reformatted section) of a bicornuate uterus. Note: The complete division of the uterine cavity and concave shape of the uterine fundus. Since fundal cleft is more than $1 \mathrm{~cm}$, this uterine anomaly is defined as a bicornuate uterus. A bicornuate uterus (commonly referred as 'heart-shaped' uterus) results from a partial nonfusion of the müllerian ducts. Finding was confirmed at the time of hysterolaparoscopic procedure

\section{Case 5}

A 34-year-old female (G2P0020) with history of dysmenorrhea and chronic pelvic pain, presents to your office with secondary infertility and history of two spontaneous abortions at 7 and 8 weeks' gestation. She is sexually active in a monogamous relationship with her husband. Onset of menarche was at 14 years. She has no history of sexually transmitted diseases. Her menstrual cycles are irregular (24-35 day cycle). The patient's history is significant for primary amenorrhea and removal of

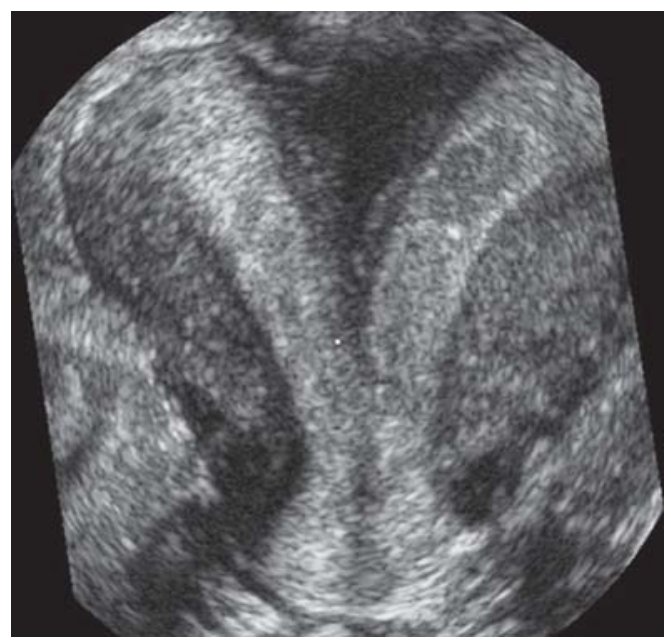

Fig. 6: Three-dimensional ultrasound image of a didelphys uterus. Duplication of the cervix and the vagina is clearly visualized. Didelphys uterus results from a complete nonfusion of both müllerian ducts. The individual horns are fully developed and almost normal in size. Two cervices are inevitably present. A longitudinal or transverse vaginal septum may also be noted. Didelphys uteri have the highest association with transverse vaginal septa, but septa also may be observed in other anomalies 
transverse vaginal septum. She is not taking any medications. Vital signs are normal. Pelvic examination revealed two cervices. The rest of the physical examination was normal. Figure 6 reveals her ultrasound findings.

\section{Case 6}

A 27-year-old female (GOPO) with history of dysmenorrhea, comes to your office for assessment of her primary dysmenorrhea. She is currently sexually active and is using oral contraceptive pills. Onset of menarche was at 15 years and she has no history of sexually transmitted diseases. Her menstrual cycles are irregular (24-50 days) with periods 6 to 8 days of duration. The patient has no significant past medical history. Her vital signs are blood pressure $128 / 80$, pulse 90 beats per minute, respiratory rate 22 breaths per minute and temperature is $37^{\circ} \mathrm{C}\left(98.6^{\circ} \mathrm{F}\right)$. Her physical examination was normal. Figure 7 displays the patient's ultrasound findings.

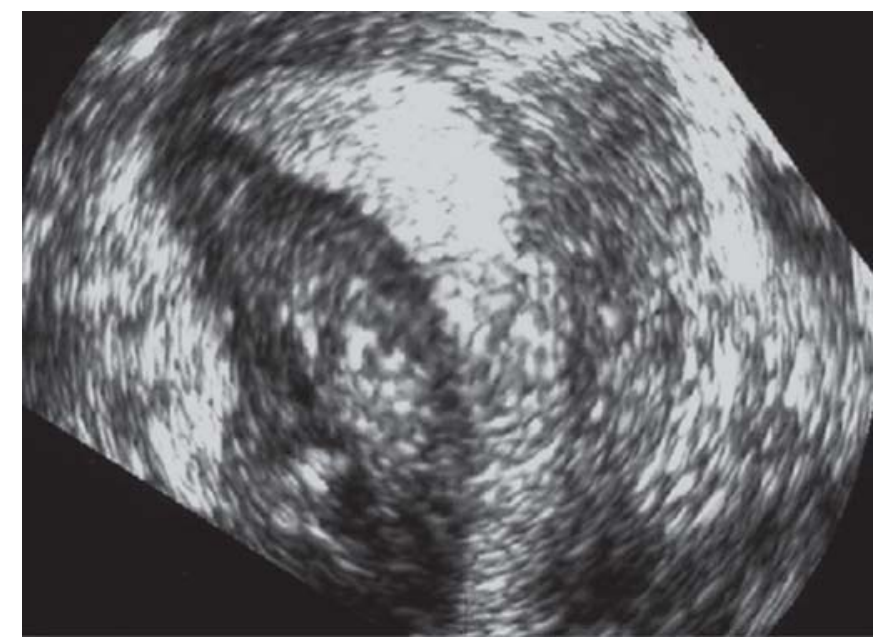

Fig. 7: Three-dimensional ultrasound image of unicornuate uterus. Unicornuate uterus develops due to the unilateral müllerian duct agenesis. Laparoscopy and hysteroscopy are required to differentiate between the different types of unicornuate uterus (with communicating rudimentary horn containing the functional endometrium, with noncommunicating rudimentary horn containing the functional endometrium, with noncommunicating rudimentary horn containing the nonfunctional endometrium and the unicornuate uterus with no evidence of rudimentary horn)

\section{DISCUSSION}

Generally, it is difficult to ascertain the true prevalence of congenital uterine anomalies because they are usually asymptomatic. Clinically, they are associated with infertility, recurrent pregnancy loss, spontaneous abortions, fetal intrauterine growth retardation, preterm births, abnormal fetal presentations and retained products of conception. The A merican Fertility Society (AFS) classification scheme categorized the congenital uterine anomalies into seven classes. Class I is uterine/cervical agenesis, class II is unicornuate uterus, class III is didel phic uterus, class IV is bicornuate uterus, class $\mathrm{V}$ is septate uterus, class VI is arcuate uterus and class VII is diethylstilbestrol-related anomaly.

As shown in Table 1, these abnormalities occur at different stages during the embryological development of the uterus. The paramesonephric (müllerian) ducts are precursors to the uterus, the cervix, the upper two-thirds of the vagina and the fallopian tubes. Organogenesis of these structures is the first stage of uterus development. Problems at this stage lead to formation of unicornuate uterus. Then lateral fusion of the paramesonephric ducts occurs in the 7th to 9th weeks of development. Improper lateral fusion leads to bicornuate uterus and didelphic uterus. During this stage, a midline septum forms in the uterine cavity and it gets resorbed within 20 weeks. Failure of the septal resorption causes arcuate uterus and septate uterus.

\section{Case 1: Arcuate Uterus}

A rcuate uterus has a single uterine cavity and it is characterized by thickened fundal myometrium, leading to a slight indentation of the uterine cavity (Fig. 8). ${ }^{6}$ The outer surface of the uterus is convex or flat. Clinically, the patient is often asymptomatic with no gynecological complications. According to a prospective study done by Jayaprakasan et $\mathrm{al}^{2}$ arcuate uterus was the most common finding in their group of 1385 subjects. A rcuate uterus had a prevalence of $11.8 \%$. It belongs to class VI in the AFS classification.

Table 1: Embryologic stages of development and the associated congenital uterine anomalies

\begin{tabular}{lll}
\hline Embryological stages & Description & Associated anomalies \\
\hline Organogenesis & $\begin{array}{l}\text { Development and formation of the } \\
\text { paramesonephric ducts-precursor to } \\
\text { cervix, uterus, fallopian tubes and } \\
\text { upper } 2 / 3 \text { of vagina } \\
\text { Lateral fusion of the paramesonephric ducts } \\
\text { in } 7 \text { to } 9 \text { weeks of development }\end{array}$ & - Unicornuate uterus \\
Fusion & Bicornuate uterus \\
Septal resorption & fusion and it regresses in 20 weeks & - Didelphic uterus \\
& Uterus duplex
\end{tabular}




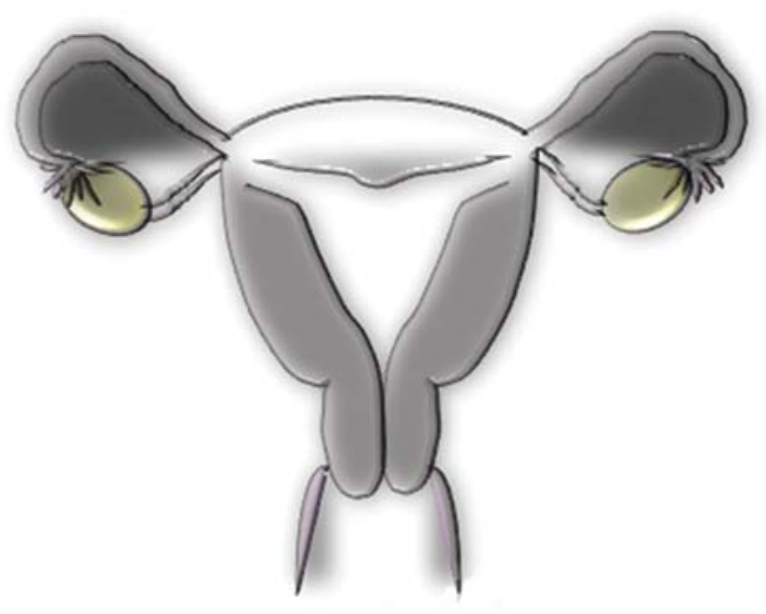

Fig. 8: Note the convex external uterine contour and a slight indentation of the uterine cavity. Arcuate uterus develops due to the failure of resorption of the midline uterine septum ${ }^{6}$ (Courtesy: Figure 19.7.23)

Unlike the other congenital uterine anomalies, it is usually associated with a normal pregnancy progression, so it is considered a normal variant. ${ }^{5}$

Between the 7th and 9th weeks of embryological development of the uterus, cervix, and the upper part of the vagina, there is fusion of the paramesonephric ducts laterally. A fter the lateral fusion occurs, there is a septum in the midline of the uterus that should ideally regress in 20 weeks. In cases like the arcuate uterus, there is minor persistence of this septum inside the uterus but most of the septum has successfully regressed. ${ }^{7}$

Standard work-up of this condition involves 2D and 3D ultrasound, saline infusion sonogrpahy (SIS) and magnetic resonance imaging (MRI). Other options involve fluoroscopy-guided hysterosal pingography (X-ray HSG) to visualize the uterine cavity, but it has lower specificity than the other options. ${ }^{6}$

Because the arcuate uterus is rarely associated with obstetric and/or gynecological complications, treatment is not usually indicated. Surgery is not indicated because it does not improve the outcome of pregnancy in women with this diagnosis. If the patient is having trouble with fertility, then she can explore assisted reproductive technology treatment options.

\section{Cases 2 and 3: Septate Uterus}

Septate uterus involves a partial or full midline septum between the two uterine horns. It may be composed of myometrium or fibrous tissue. ${ }^{7} \mathrm{U}$ sing $3 \mathrm{D}$ ultrasound, septate uterus is diagnosed in coronal plane, with a septum producing a sagittal notch in the uterine cavity more than equal to $1 \mathrm{~cm}$. Similar to the arcuate uterus, the external contour of the uterus is convex or flat. Septate uterus can

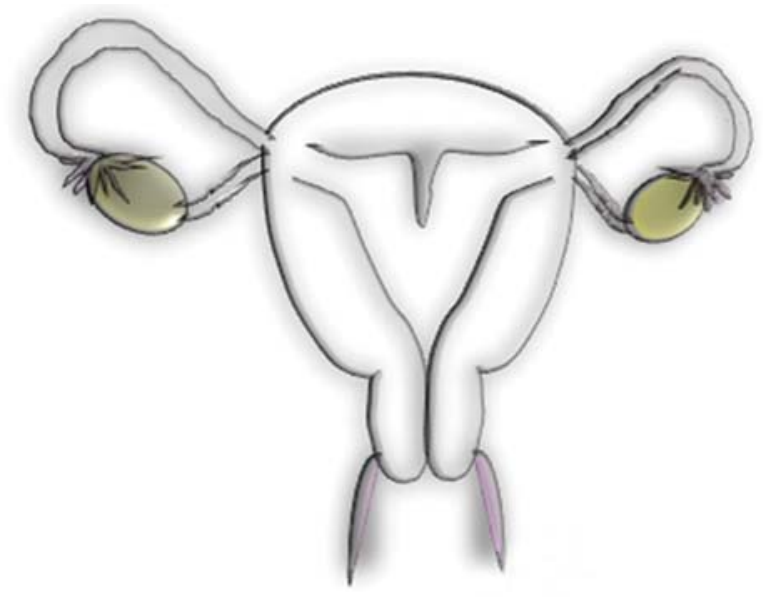

Fig. 9: Partial septate uterus results from partial failure of resorption of the uterovaginal septum after fusion of the paramesonephric ducts $^{6}$ (Courtesy: Figure 19.7.27)

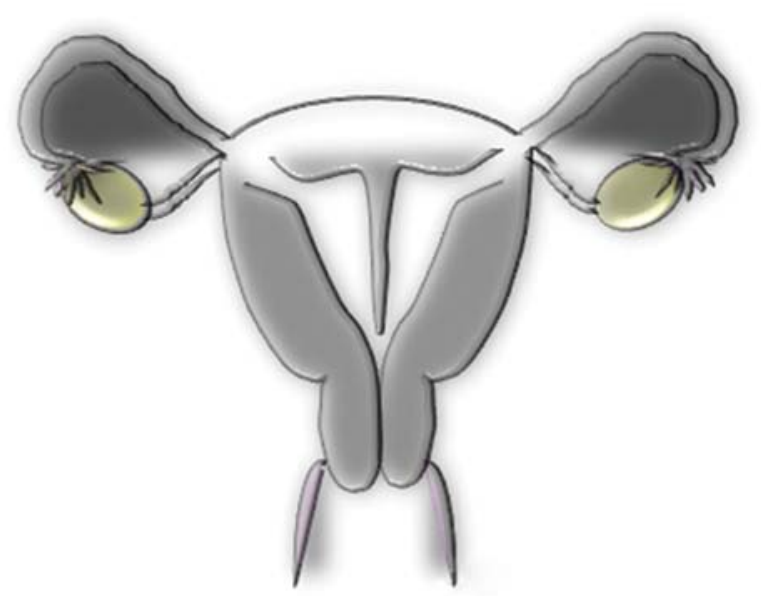

Fig. 10: Complete uterine septum ${ }^{6}$ (Courtesy: Figure 19.7.31)

be further divided into the partial (or incomplete) and complete (Fig. 9). ${ }^{6} \mathrm{~A}$ full septum can extend as far as into the internal cervical os (Fig. 10). Septate uterus may be clinically asymptomatic until pregnancy or patient may report episodes of dysmenorrhea. It is associated with high incidence of spontaneous abortion, miscarriages and premature birth. It is categorized as class V in AFS. It is reported to have $0.5 \%$ prevalence. It is also more clinically complicated than the arcuate uterus, because patients with the septate uterus have the highest incidence of reproductive difficulties.

Embryologically, the septate uterus results from an abnormality of the midline septal regression (similar to the development of the arcuate uterus) after complete fusion of the müllerian ducts. ${ }^{5}$

Work-up options are standard with 2D and 3D ultrasound, SIS, X-ray HSG and M RI. Hysteroscopy and laparoscopy allow direct visualization of the intrauterine 
and abdominal cavity. ${ }^{8}$ It is therefore, considered a 'gold standard' to establish a definitive diagnosis and treat the patient with different types of uterine anomalies (Table 2). Table 2 reviews advantages and disadvantages of different diagnostic procedures. Treatment options involve surgical resection of the septum via transvaginal hysteroscopyusually over $90 \%$ of the septum is removed. ${ }^{3,9}$ The transcervical approach to hysteroscopic metroplasty is superior to the abdominal approach due to lower morbidity rates and intra-abdominal adhesions that may cause fertility problems later on. Imaging work-up should be done around 2 months after surgery for follow-up to assess successful healing. If the septum cannot be safely removed hysteroscopically, then other methods are laparascopic/ abdominal approaches, like the Jones or Tompkins metroplasty. J ones metroplasty involves a triangular, wedgeshaped resection of the section of the uterus containing the septum for complete septal removal. Tompkins metroplasty does not involve any tissue removal but they dissect and combine the separated uterine cavities, resulting in a much larger one. ${ }^{3}$

K upesic and $\mathrm{K}$ urjak ${ }^{8}$ studied the incidence of surgically correctable uterine abnormalities (congenital uterine anomalies, submucous leiomyoma, endometrial polyps and intrauterine synechiae) in the infertile population attending a tertiary infertility clinic. A II the infertile patients enrolled in the study were evaluated by $3 \mathrm{D}$ ultrasound. ${ }^{9,10}$ A nother objective of these studies was to assess pregnancy rates before and after operative hysteroscopy in patients affected by uterine causes of infertility. The prevalence of uterine abnormalities in subfertile population was $7.9 \%$. The most common uterine abnormality accounting for $77.1 \%$ of the uterine causes of infertility was the septate uterus. O ut of 310 patients who were followed-up, $225(72.6 \%)$ patients achieved pregnancy after hysteroscopic metroplasty for an intrauterine septum. ${ }^{11,12}$

In patients with recurrent abortions and septate uterus, reproductive outcome was significantly improved after

Table 2: Diagnostic procedures for detection of congenital uterine anomalies

\begin{tabular}{|c|c|c|}
\hline Diagnostic method & Advantages & Disadvantages \\
\hline X-ray HSG & $\begin{array}{l}\text { - Provides a good outline of the } \\
\text { uterine cavity }\end{array}$ & $\begin{array}{l}\text { - Invasive } \\
\text { - Exposure to radiation } \\
\text { - } \text { Lack of visualization of the uterine fundus } \\
\text { - Impossible for detection of minor anomalies } \\
\text { types of fusion and resorption disorders }\end{array}$ \\
\hline 2D ultrasound & $\begin{array}{l}\text { - Noninvasive } \\
\text { - Uterine cavity can be visualized in } \\
\text { longitudinal and transverse planes } \\
\text { Detection of the two endometrial echoes } \\
\text { in the upper portion of the uterine cavity } \\
\text { in transverse plane indicates duplication } \\
\text { anomaly of the uterus } \\
\text { - Good screening tool }\end{array}$ & $\begin{array}{l}\text { - Does not visualize coronal plane } \\
\text { - Morphology of the uterus cannot } \\
\text { be objectively analyzed }\end{array}$ \\
\hline 3D ultrasound & $\begin{array}{l}\text { - Noninvasive } \\
\text { - } \text { Visualization of the coronal plane } \\
\text { Assessment of planar reformatted } \\
\text { - Surface rendering of the uterine cavity } \\
\text { - Simultaneous assessment of the fundal shape }\end{array}$ & $\begin{array}{l}\text { - Lack of availability and expertise } \\
\text { - Operator dependent } \\
\text { - Requires technical competence } \\
\text { Limitations: Shadowing caused by the } \\
\text { uterine fibroids, irregular endometrial lining } \\
\text { and decreased volume of the uterine cavity } \\
\text { (in cases of intrauterine adhesions) }\end{array}$ \\
\hline Saline infusion sonography & $\begin{array}{l}\text { - Invasive } \\
\text { - In the same time may demonstrate } \\
\text { tubal patency }\end{array}$ & $\begin{array}{l}\text { - Minimally invasive } \\
\text { - Operator dependent } \\
\text { - Avoids radiation exposure } \\
\text { - Patients' discomfort (uterine contractions) }\end{array}$ \\
\hline MRI & $\begin{array}{l}\text { - Noninvasive } \\
\text { - Simultaneous visualization of the } \\
\text { uterine cavity and fundal shape } \\
\text { - High diagnostic precision and accuracy }\end{array}$ & - Expensive \\
\hline Hysteroscopy & - Can identify the presence of an anomaly & $\begin{array}{l}\text { - Invasive } \\
\text { - Cannot accurately differentiate between the } \\
\text { different subtypes of congenital uterine } \\
\text { anomalies }\end{array}$ \\
\hline Hysteroscopy and laparoscopy & - Allow concurrent diagnosis and treatment & - Invasive \\
\hline
\end{tabular}

2D: Two-dimensional; 3D: Three-dimensional; HSG: Hysterosalpingography; MRI: Magnetic resonance imaging; SIS: Saline infusion sonography 
metroplasty. ${ }^{13-15}$ Higher risk of miscarriage was reported among patients incidentally diagnosed with septate uterus who conceived after medically assisted reproduction. ${ }^{2}$ In this respect, infertile patients undergoing assisted reproduction techniques should undergo detailed assessment of uterine morphology by 3D ultrasound. G hi et al ${ }^{16}$ reported that the women incidentally diagnosed with septate uterus during their first pregnancy have an extremely high risk of miscarriage, either in their first or second trimester.

\section{Case 4: Bicornuate Uterus}

Bicornuate uterus (commonly referred as 'heart-shaped' uterus) results from a partial nonfusion of the müllerian ducts (Fig. 11). ${ }^{6}$ In complete bicornuate uterus the central myometrium may extend to the level of the internal cervical os (bicornuate unicollis) or external cervical os (bicornuate bicollis). The latter is distinguished from the uterus didel phic because it demonstrates some degree of fusion betw een the two horns, while in classic didelphic uterus, the two horns and cervices are completely separated. A nother difference between dydelphic and bicornuate uterus is that the horns of the bicornuate uteri are not fully developed; typically, they are smaller than those of didelphic uteri. It is class IV of AFS. Prevalence was reported to be $0.1 \%$ of the general population. Clinically, the pregnancy rates of patients with bicornuate uteri are similar to the general population, so surgery is not usually indicated. It is an option if the patient has a history of recurrent pregnancy loss. Reunification of the uterus is an option via laparoscopy or laparotomy.

This abnormality occurs in the 7th to 9th weeks of embryological development when the paramesonephric ducts fuse to form the uterus in the initial stages. The

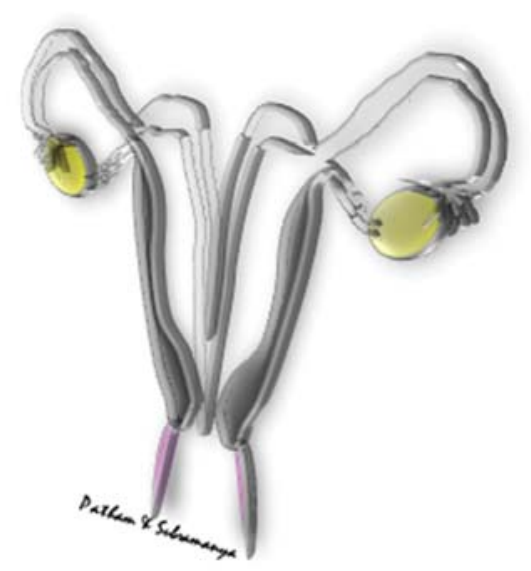

Fig. 11: Schematic image of bicornuate uterus. Note: The clear separation of the uterine cavity and fundal indentation ${ }^{6}$ (Courtesy: Figure 19.7.34) paramesonephric ducts grow laterally to the urogenital ridges. In this stage of development, there is lateral fusion of the paramesonephric ducts which ultimately becomes the uterus, cervix, the fallopian tubes and the upper two-thirds of the vagina. Bicornuate uterus forms as a result of improper fusion of the paramesonephric ducts and the horns of the uterus are not fully developed., 6,7

\section{Case 5: Didelphic Uterus}

Didelphic uterus is comprised of two separate, fullydeveloped uterine horns with two cervices (Fig. 12). It belongs to class III of AFS. It has a high association with transverse vaginal septa. This may lead to difficulties in intercourse or vaginal delivery. Clinically, patients may present with preterm births, recurrent pregnancy loss and pelvic pain. Patients may opt to have the septum resected and have metroplasty performed. ${ }^{3,13}$ These patients have good reproductive outcomes and they have full-term pregnancies because each horn is technically a fullydeveloped uterus. Surgical treatment is not indicated if a patient has recurrent pregnancy loss, because it is generally believed that it would not improve pregnancy outcomes. ${ }^{13}$

Embryologically, didel phic uterus has a similar etiology as bicornuate uterus. It is due to failure of fusion of the paramesonephric ducts during the 7th to 9th weeks of development.

\section{Case 6: Unicornuate Uterus}

U nicornuate uterus has a rudimentary horn that may or may not have functioning endometrium, therefore, half of the uterus is not fully-developed (Fig. 13). I t is reported to have

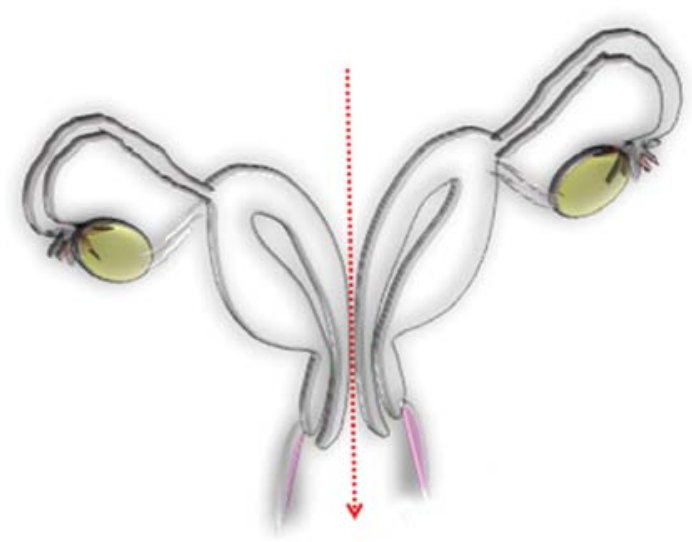

Fig. 12: Didelphic uterus results from a complete nonfusion of both müllerian ducts. The individual horns are fully developed and almost normal in size. Two cervices are inevitably present. A longitudinal or transverse vaginal septum may also be noted ${ }^{6}$ (Courtesy: Figure 19.7.36) 


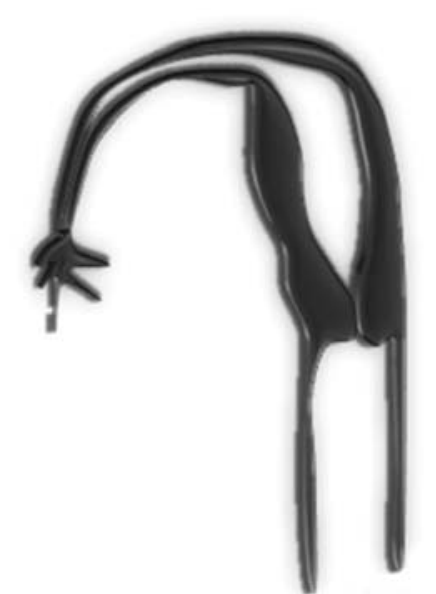

Fig. 13: Schematic image of unicornuate uterus with no evidence of rudimentary horn ${ }^{6}$ (C ourtesy: Figure 19.7.36)

a prevalence of $0.4 \%$ in the general population, but it is more common in infertile women. It is classified as class II in the AFS. ${ }^{5}$ If there is obstruction present in the rudimentary horn with functional endometrium, then the patient would present with a pelvic mass, chronic abdominal/pelvic pain and they may require surgical excision of the rudimentary horn laparoscopically. Surgical excision is imperative because the obstructed rudimentary horn places the patient at higher risk of endometriosis, and its removal prevents future occurrence because of no reflux. ${ }^{3}$ Clinically, unicornuate uterus is associated with spontaneous abortion, ectopic ovaries, endometriosis, preterm delivery, ectopic pregnancy and kidney abnormalities. Generally, surgery is not indicated for the repair of unicornuate uteri because it is not believed to improve pregnancy outcomes.

Embryologically, unicornuate uterus develops because of incomplete development of one of the paramesonephric ducts, leading to an asymmetric lateral fusion defect.

\section{CONCLUSION}

A number of imaging techniques can be used to diagnose and differentiate among congenital uterine anomalies. 2D ultrasound has low accuracy rates, but can be efficiently used as screening tool. X-ray HSG and SIS are highly accurate for the assessment of the uterine cavity, but are invasive and do not clearly demonstrate the shape of the uterine fundus. N umerous studies suggest that 3D ultrasound is very accurate for the assessment of the uterine cavity and fundal shape, but at this moment has limited availability. Due to high cost, MRI is not accepted as screening or diagnostic tool for detection of congenital uterine anomalies. Furthermore, more studies are required to determine its diagnostic accuracy. By many specialists, hysteroscopy and laparoscopy is considered the 'gold standard', which allows direct visualization of the uterine cavity and treatment of the uterine defect. Clearly, more studies are needed to provide clear guidelines regarding clinical implications of congenital uterine anomalies detected by different imaging methods on pregnancy outcome.

\section{REFERENCES}

1. Shipp TD. Ultrasound examination in obstetrics and gynecology. U ptodate 2012. [A ccessed J uly, 9 2012]. http://www.uptodate. com/contents/basic-principles-and-safety-of-diagnosticultrasound-in-obstetrics-and-gynecology.

2. Jayaprakasan J, Chan YY, Sur S, et al. Prevalence of uterine anomalies and their impact on early pregnancy in women conceiving after assisted reproduction treatment. U Itrasound Obstet Gynecol 2011;37(6):727-32.

3. I verson RE, DeC herney A H, L aufer M R. Clinical manifestations and diagnosis of congenital anomalies of the uterus. U ptodate2011. http://www .uptodate.com/contents/clinicalmanifestations-and-diagnosis-of-congenital-anomalies-of-theuterus.

4. Bermejo C, M artinez TP, Cantarero R, D iaz D, Perez Pedregosa $J, B$ arron $\mathrm{E}$, et al. Three-dimensional ultrasound in the diagnosis of Mullerian duct anomalies and concordance with magnetic resonance imaging. Ultrasound Obstet Gynecol 2010;35: 593-601.

5. Syed I. Imaging in Müllerian Duct Abnormalities. M edscape2011. http://emedicine.medscape.com/article/405335overview.

6. K upesic PS. Pelvic ultrasound pearls. In: K upesic PS (Ed). Color Doppler, 3D and 4D U Itrasound in Obstetrics, Gynecology and Infertility. London, N ew D elhi, Panama City, St. L ouis: J aypee Publishers 2011;256-348.

7. Healey A. Imaging of gynecological disorders in infants and children. Embryology of the Female R eproductive Tract. B erlin: Springer-V erlag B erlin Heidel berg 2012.

8. K upesic $S, K$ urjak A. Diagnosis and treatment outcome of the septate uterus. Croatian M ed J 1998;39:185-90.

9. Kupesic S. Clinical implications of sonographic detection of uterine anomal ies for reproductive outcome. U Itrasound 0 bstet Gynecol 2001;18(4):387-400.

10. K upesic $S, K$ urjak A. Role of three-dimensional ultrasound in diagnosis of congenital uterine anomalies. Ultrasound Rev Obstet Gynecol 2005;5:194-201.

11. Sparac V, Kurjak A, Kupesic S, Goldenberg M, Ilijas M, Skenderovic $S$, et al. Histological architecture and vascularization of intrauterine septa excised during hysteroscopic procedure. Gynecol Perinatol 1996;5:101-03.

12. K upesic S, Kurjak A, Skenderovic S, Bjelos D. Screening for uterine abnormalities by three-dimensional ultrasound improves perinatal outcome. J Prenat M ed 2002;30:9-17.

13. Chan $Y Y$, Jayaprakasan $K, \operatorname{Tan} A$, Thornton J G, Coomarasamy A, Raine-Fenning NJ. Reproductive outcome in women with congenital uterine anomalies: A systematic review. Ultrasound Obstet Gynecol 2011;38:371-82. 
14. Mollo A, De Franciscis $P$, Colacurci N, Cobellis L, Perino A, $V$ enezia $R$, et al. Hysteroscopic resection of the septum improves the pregnancy rate of women with unexplained infertility: A prospective controlled trial. Fertil Steril 2009;91:2628-31.

15. Pabuccu R, Gomel V. Reproductive outcome after hysteroscopic metroplasty in women with septate uterus and otherwise unexplained infertility. Fertil Steril 2004;81:1675-78.

16. Ghi T, De M usso F, M aroni E, Y oussef A, Savelli L, Farina A, et al. The pregnancy outcome in women with incidental diagnosis of septate uterus at first trimester scan. Hum Reprod 2012 Sep;27(9):2671-75.

\section{ABOUT THE AUTHORS}

\section{Sarah Tabi}

Student Physician, SUNY U pstate COM, Clinical Campus at Binghamton, N ew Y ork, USA

\section{Sanja Kupesic Plavsic (Corresponding Author)}

Professor, D epartment of Obstetrics/G ynecology and R adiology, Paul L Foster School of M edicine, Texas Tech University, 4800 A Iberta A ve, EI Paso, Texas 79905, USA, Phone: $+1(915) 783-1700$ e-mail: sanja.kupesic@ttuhsc.edu

\section{SELF-ASSESSMENT QUIZ}

1. For which of the congenital uterineanomalies listed below surgery is not indicated?
A. Arcuate uterus
B. Septate uterus
C. Bicornuate uterus
D. Didelphic uterus
E. A, C, and D

2. Which of the congenital uterine anomalies is a result of failure of lateral fusion of paramesonephric ducts?
A. Bicornuate uterus
B. Didelphic uterus
C. Both
D. N either

3. Which congenital uterineanomaly is not associated with any obstetric or gynecological complication?
A. Septate uterus
B. Unicornuate uterus
C. A rcuate uterus
D. Didelphic uterus

4. Why aremüllerian duct anomalies underreported?
A. M any affected patients are asymptomatic
B. There are not sufficient imaging modalities available to detect congenital uterine anomalies
C. Not all types of müllerian duct anomalies have been discovered yet
D. None of the above
E. $A$ and $B$

5. Which congenital uterine anomaly has the highest risk of pregnancy complications?
A. A rcuate uterus
B. Septate uterus
C. Didelphic uterus
D. Bicornuate uterus

6. What is a common complication of didelphic uterus?
A. Pelvic inflammatory diseas
B. Prolapsed uterus
C. Endometriosis
D. Transverse vaginal septum

7. What is prevented by doing a surgical excision of an obstructed rudimentary horn of a unicornuate uterus?
A. Infection
B. Uterine cancer
C. Endometriosis
D. All of the above

8. Why is 3D ultrasound superior to 2D ultrasound?
A. It detects inconspicuous uterine anomalies
B. It images the internal and external contours of the uterus with ease
C. Displays the coronal plane of the uterus with a sharper image
D. All of the above

9. What are the limitations of 3D ultrasound?
A. It has a high rate of false positive results
B. There is difficulty imaging uteri with endometrium less than $5 \mathrm{~mm}$ thick
C. There is a high risk of cancer as a side effect of this imaging modality
D. None of the above

10. What is reported to be the most common müllerian duct anomaly?
A. Septate uterus
B. Didelphic uterus
C. Arcuate uterus
D. Bicornuate uterus

(1) E; (2) C; (3) C; (4) E; (5) B; (6) C; (7) D; (8) D; (9) B; (10) C 